\title{
Impacto da Doença de Alzheimer na qualidade de vida de pessoas idosas: revisão de literatura
}

\author{
Impact of Alzheimer's disease in the quality of life of elderly: literature review
}

Impacto de la enfermedad de Alzheimer en la calidad de vida de las personas mayores: revisión de la literatura

Tamiris de Queiroz Rodrigues ${ }^{1}$, Alice da Silva de Castro ${ }^{1}$, Tifany Fernandes da Conceição ${ }^{1}$, João Guilherme Alves Moraes Leite ${ }^{1}$, Vitor Hugo Sales Ferreira ${ }^{1}$, Andréa Mathes Faustino ${ }^{1 *}$.

\section{RESUMO}

Objetivo: Analisar o impacto que a Doença de Alzheimer tem sobre a qualidade de vida do idoso. Métodos: Trata-se de uma revisão integrativa da literatura, na qual o levantamento bibliográfico foi realizado em bases de dados nacionais e internacionais. Foram utilizados os descritores em saúde: Idoso, Qualidade de Vida, Doença de Alzheimer. Fizeram parte da amostra final 9 artigos que se encaixavam nos critérios de inclusão. Resultados: Os artigos foram agrupados em duas classes de assuntos gerais: Aspectos fisiopatológicos e epidemiológicos da Doença de Alzheimer; e Qualidade de vida e sua relação com a família e o cuidador de idosos com Doença de Alzheimer. Os fatores encontrados que culminaram na menor qualidade de vida dos idosos com Doença de Alzheimer estão relacionados a disfunção cognitiva e a perda de memória, que de modo progressivo compromete a autonomia das atividades diárias básicas. Considerações Finais: Frente a prevalência das doenças neurodegenerativas progressivas acometidas na população idosa foi possível observar que ocorre uma redução expressiva da qualidade de vida dos idosos portadores da Doença de Alzheimer, relacionadas sobremaneira, a perda de autonomia e dependência em suas atividades diárias.

Palavras-chave: Qualidade de vida, Doença de Alzheimer, Idoso, Expectativa de vida.

\section{ABSTRACT}

Objective: To analyze the impact that Alzheimer Disease has in the quality of life of the elderly. Methods: This is an integrative literature review study, in which the bibliographic survey was carried out in national and internacional databases. The keywords used in health were: Elderly, Quality of Life, Alzheimer's Disease. The final sample included 9 articles that met the inclusion criteria. Results: The articles were grouped into two general subject classes: Pathophysiological and epidemiological aspects of Alzheimer's Disease; and Quality of life and its relationship with the family and caregiver of elderly people with Alzheimer's disease. The factors that culminated in the lower quality of life of elderly people with Alzheimer's disease are related to cognitive dysfunction and memory loss, which progressively compromises the autonomy of basic daily activities. Final Considerations: Given the prevalence of progressive neurodegenerative diseases in the elderly population, it was possible to observe that there is a significant reduction in the quality of life of elderly people with Alzheimer's Disease, mainly related to the loss of autonomy and dependence on their daily activities.

Key words: Quality of life, Alzheimer's disease, Seniors, Life expectancy.

${ }^{1}$ Departamento de Enfermagem, Faculdade de Ciências da Saúde, Universidade de Brasília, Brasília - DF. *E-mail: andreamathes@unb.br

SUBMETIDO EM: 12/2019

ACEITO EM: 1/2020

PUBLICADO EM: 3/2020 


\section{RESUMEN}

Objetivo: analizar el impacto que tiene la Enfermedad de Alzheimer en la calidad de vida de los ancianos. Métodos: Este es un estudio integrador de revisión de literatura, en la cual la encuesta bibliográfica se realizó en bases de datos nacionales e internacionales. Las palabras clave utilizadas en salud fueron: Ancianos, Calidad de vida, Enfermedad de Alzheimer. La muestra final incluyó 9 artículos que cumplieron con los criterios de inclusión. Resultados: Los artículos se agruparon en dos clases de materias generales: aspectos fisiopatológicos y epidemiológicos de la enfermedad de Alzheimer; y Calidad de vida y su relación con la familia y el cuidador de personas mayores con enfermedad de Alzheimer. Los factores que culminaron en la baja calidad de vida de las personas mayores con enfermedad de Alzheimer están relacionados con la disfunción cognitiva y la pérdida de memoria, que compromete progresivamente la autonomía de las actividades diarias básicas. Consideraciones finales: Dada la prevalencia de enfermedades neurodegenerativas progresivas en la población de edad avanzada, fue posible observar que existe una reducción significativa en la calidad de vida de las personas de edad avanzada con enfermedad de Alzheimer, principalmente relacionada con la pérdida de autonomía y la dependencia de sus actividades diarias.

Palabras clave: Calidad de vida, Enfermedad de Alzheimer, Anciano, Expectativa de vida.

\section{INTRODUÇÃO}

O crescimento da população de idosos é um fenômeno mundial, as projeções indicam que em 2050, o Brasil tenha uma população de 253 milhões de habitantes. A estimativa para o ano de 2040 , é que este número dobre, representando $23,8 \%$ da população brasileira total. Essa nova realidade demográfica, com um número cada vez maior de idosos, vai exigir mais do sistema de saúde para responder às demandas atuais e futuras. No ano de 2010, havia no Brasil cerca de 20,5 milhões de idosos, aproximadamente 39 para cada grupo de 100 jovens. (MIRANDA GMD, et al., 2016).

Com a transição epidemiológica que ocorre no Brasil houve um aumento da prevalência de doenças crônicas não transmissíveis (DCNT) em detrimento das doenças infectocontagiosas, devido a melhora nas condições econômicas, sociais e culturais do país. Tais melhorias resultaram no aumento da expectativa de vida, que por sua vez culminou no predomínio de morbidades progressivas, sem possibilidade de cura e que são influenciadas por fatores genéticos, comportamentais, de idade e gênero (HOLANDA ITA, et al., 2012).

À medida que a expectativa de vida se torna mais elevada, pode ser observado um aumento da prevalência das demências, sendo a de maior ocorrência a Doença de Alzheimer (DA). O curso da doença pode variar entre 5 e 10 anos, além disto a expectativa de vida pode reduzir em 50\% (FERNANDES JSG, ANDRADE MS, 2017).

Até 2050, espera-se que o número de pessoas que vivem com demência triplique, de 50 milhões para 152 milhões. $E$ entre as pessoas que desenvolvem Doença de Alzheimer, quase seis milhões delas vivem em países de baixa e média renda. O custo anual com a DA é estimado em US $\$ 818$ bilhões, o que equivale a cerca de mais de $1 \%$ do produto interno bruto no mundo, sendo estes custos diretamente relacionados aos custos médicos, assistência social e cuidados informais (perda de renda dos cuidadores) (OPAS, 2017).

A incidência da DA é cerca $3 \%$ dos indivíduos que se encontram na faixa etária entre 60 e 65 anos, quando observada a população com 80 anos ou mais, este contingente atinge $30 \%$ destes indivíduos, em âmbito mundial e no Brasil (MIRANDA GMD, et al., 2016).

Sendo caracterizada por ser uma doença neurológica degenerativa, progressiva e irreversível, que além de deteriorar progressivamente o nível cognitivo do indivíduo, gradativamente atinge também a capacidade funcional, levando-o a desenvolver maior dependência para o autocuidado, e assim a necessidade de um cuidador que possa auxiliar em suas atividades e com o progresso da doença há a necessidade de maior nível de cuidados, o que pode trazer forte impacto tanto na vida do paciente com DA quanto para as pessoas ao seu redor e de maior convivência (FERNANDES JSG e ANDRADE MS, 2017). 
As alterações físicas e psíquicas decorrentes da doença geram situações estressoras que interferem nos relacionamentos familiares com a pessoa idosa. O afastamento da família, a sobrecarga nos cuidados e a redução da qualidade de vida daqueles que zelam pelo paciente são fatores preponderantes para reduzir sobremaneira a qualidade de vida da pessoa doente. A perda da capacidade de reconhecer os familiares e a dependência total para as atividades básicas da vida cotidiana são citados na literatura como uma das maiores dificuldades que os familiares apresentam nos cuidados dos idosos portadores de Alzheimer.

A falta de preparo e principalmente de conhecimento acerca do curso progressivo da doença são fatores que interferem diretamente no vínculo familiar e nos cuidados prestados a esses idosos (BORGHI AC, et al., 2011). Por ser uma doença neurodegenerativa progressiva e irreversível que se manifesta por deterioração cognitiva e perda de memória, ocorrem alterações comportamentais que interferem na autonomia do indivíduo portador em realizar atividades cotidianas, além de estreitar suas relações interpessoais.

Diante disso o idoso torna-se dependente de outra pessoa para cuidados básicos, de modo a suprir sua incapacidade funcional e assim pode afetar sua qualidade de vida (BORGHI AC, et al., 2011). Embora tenha ocorrido um aumento da expectativa de vida, o mesmo não acompanhou melhorias para a qualidade de vida de idosos com DA. Tal fato está associado a elevada incidência de doenças neurodegenerativas que compromete a autonomia e o bem-estar do indivíduo, como a DA (DAWALIBI NW, et al., 2014).

Frequentemente, a redução da autonomia provocada pela DA suscita um julgamento por parte dos familiares e comunidade de que o idoso se tornou incapaz de tomar decisões, porém sabe-se que é essencial manter um mínimo grau de independência nas atividades diárias básicas destes idosos, possibilitando que o mesmo execute os afazeres nos quais ainda possui capacidade, estimulando suas funções cognitivas e motoras até quando forem possíveis de serem realizadas (FERREIRA OGL, et al., 2012).

A definição de qualidade de vida (QV) pela Organização Mundial de Saúde (OMS) é aquela que descreve como a percepção do indivíduo de sua posição na vida, tendo que ser observada em vários contextos, tais como de cultura e sistemas de valores nos quais o indivíduo está inserido, expectativas pessoais, padrões e preocupações, sendo assim um conceito multidimensional o qual deve ser tratado em suas várias dimensões: social, mental, material, física, cultural, econômica (INOUYE K, et al., 2010).

No contexto das famílias que assumem o cuidado da pessoa com DA, geralmente esta tarefa é delegada a uma pessoa que possui outras tarefas e necessita conciliá-las, e na maioria das vezes é realizada por uma mulher (filha, esposa, irmã). O acúmulo de tarefas e papéis sociais e familiares, exercidos pelo cuidador impactam diretamente em sua saúde, e assim consequentemente em sua QV, não podendo ofertar muitas vezes um cuidado com qualidade, devido à sobrecarga a que está exposto, o que irá manifestar também na QV do idoso a que está submetido aos seus cuidados (PEREIRA LSM, SOARES SM, 2015).

A indicação de uma má qualidade de vida, entre cuidadores de pessoas com DA, pode estar associada à um quadro de estresse. A percepção de pior qualidade de vida entre os cuidadores familiares está intrinsecamente relacionada à devastadora experiência de cuidar, que decorrem de preocupações sobre a evolução e o prognóstico da doença, além de todas as tarefas do cuidado diário, dedicado a um idoso com DA. A tarefa de cuidar pode desencadear alterações físicas, emocionais, espirituais e sociais (CEZARIO VAC, et al., 2017).

A presente pesquisa tem por objetivo analisar, a partir de uma revisão bibliográfica, o impacto que a DA tem sobre a qualidade de vida do idoso. Por se tratar de uma enfermidade abrangente, optou-se por ressaltar os impactos relacionado aos aspectos da autonomia, saúde psicológica e vínculos familiares dos portadores de Alzheimer.

\section{MÉTODOS}

Estudo do tipo revisão integrativa da literatura. Para o procedimento de seleção dos artigos foi formulada a seguinte pergunta norteadora: "Quais são os impactos que a DA tem sobre a qualidade de vida da pessoa idosa?". Para a execução do estudo foram seguidas as etapas já descritas na literatura para a implementação 
do método de revisão integrativa da literatura: formulação do problema; coleta de dados; avaliação dos dados; análise e interpretação dos dados e divulgação dos dados (SOARES CB, et al., 2014).

Os critérios de inclusão definidos para a seleção dos artigos foram: ser artigo publicado em português, inglês ou espanhol, com os resumos disponíveis nas bases de dados para a seleção; artigos que estivessem disponíveis na íntegra com acesso on-line nas bases de dados pesquisadas, publicados no período compreendido entre os anos de 2008 a 2018 no Brasil ou na América do Sul, cuja temática principal fosse os impactos que a DA tem sobre a qualidade de vida do idoso. Foram excluídos da pesquisa, os artigos que não abordassem o tema de qualidade de vida dos idosos portadores de DA, aqueles que não estivessem disponíveis na íntegra gratuitamente para leitura ou que não contassem com a população idosa no estudo.

Para a seleção do material bibliográfico foram utilizadas as seguintes bases de dados: Medical Literature Analysis and Retrieval System on-line (MEDLINE) disponibilizada pela PUBMED da U.S. National Library of Medicine National Institutes of Health, Literatura Latino-Americana e do Caribe em Ciências da Saúde (LILACS), Base de Dados de Enfermagem (BDENF) da Biblioteca Virtual em Saúde (BVS), Scientific Electronic Library Online(SCIELO) e Google Acadêmico, que englobam as principais produções científicas da área da enfermagem. Foram também consideradas a literatura cinzenta, a qual trata-se de material de literatura não controlada por editores científicos ou comerciais. Podem ser incluídos relatórios governamentais, teses, dissertações e resumos provenientes de anais de eventos científicos (PEREIRA MG, GALVÃO TF, 2014).

Para a realização das buscas nas bases de dados foram utilizados os seguintes descritores de assunto em saúde (DECS/MESH): Aged, Quality of Life, Alzheimer Disease; Idoso, Qualidade de Vida, Doença de Alzheimer; Anciano, Calidad de Vida, Enfermedad de Alzheimer. Para análise dos artigos, foi realizada leitura na íntegra dos estudos com auxílio de instrumento específico para o método escolhido. Após essa etapa, foi realizada discussão entre o grupo de autores, sendo que as divergências foram solucionadas por meio de releitura dos estudos pelos autores. A seguir, os artigos foram categorizados segundo a temática abordada e agrupados por assunto apresentado por cada um dos autores, a fim de demonstrar os assuntos mais relevantes em cada artigo encontrado.

\section{RESULTADOS}

A amostra desta revisão foi constituída por 23 artigos que foram selecionados previamente, contudo após a leitura foram excluídos 14 artigos que não eram específicos ao tema e não se encaixavam nos critérios de inclusão estabelecidos, ficando uma amostra final de 9 artigos incluídos na revisão. O Quadro 1 apresenta os artigos selecionados e categorizados. 
Quadro 1 - Distribuição dos artigos selecionados, segundo autores, título do artigo, tipo de estudo, ano de publicação, origem do estudo e idioma, n=9.

\begin{tabular}{|c|c|c|c|c|c|}
\hline AUTORES & TÍTULO DO ARTIGO & TIPO DE ESTUDO & $\begin{array}{c}\text { ANO DE } \\
\text { PUBLICAÇÃO }\end{array}$ & $\begin{array}{l}\text { ORIGEM DO } \\
\text { ESTUDO }\end{array}$ & IDIOMA \\
\hline $\begin{array}{l}\text { Sereniki A e Vital } \\
\text { MABF }\end{array}$ & $\begin{array}{l}\text { A doença de Alzheimer: aspectos fisiopatológicos e } \\
\text { farmacológicos }\end{array}$ & Revisão literária & 2008 & Brasil & Português \\
\hline Freitas ICC, et al. & $\begin{array}{l}\text { Convivendo com portador de Alzheimer: perspectivas } \\
\text { do familiar cuidador }\end{array}$ & Descritivo & 2008 & Brasil & Português \\
\hline Vital TM, et al. & $\begin{array}{c}\text { Atividade física sistematizada e sintomas de } \\
\text { depressão na demência de Alzheimer: uma revisão } \\
\text { sistemática }\end{array}$ & $\begin{array}{l}\text { Revisão } \\
\text { sistemática }\end{array}$ & 2010 & Brasil & Português \\
\hline Inouye $\mathrm{K}$, et al. & $\begin{array}{c}\text { Influência da doença de Alzheimer na percepção de } \\
\text { qualidade de vida do idoso }\end{array}$ & Comparativo & 2010 & Brasil & $\begin{array}{l}\text { Inglês } \\
\text { português }\end{array}$ \\
\hline Holanda ITA, et al. & Idosos com Alzheimer: um estudo descritivo & $\begin{array}{l}\text { Exploratório e } \\
\text { descritivo }\end{array}$ & 2012 & Brasil & Português \\
\hline Pereira EF, et al. & Qualidade de vida: abordagens, conceitos e avaliação & Revisão literária & 2012 & Brasil & Português \\
\hline Burlá C, et al. & $\begin{array}{c}\text { Envelhecimento e reflexões sobre doença de } \\
\text { Alzheimer: reflexões sobre autonomia e o desafio do } \\
\text { cuidado }\end{array}$ & Revisão integrativa & 2014 & Brasil & Português \\
\hline $\begin{array}{l}\text { Assis CRC e Camacho } \\
\text { ACLF. }\end{array}$ & $\begin{array}{l}\text { Qualidade de vida dos idosos com doença de } \\
\text { Alzheimer: uma revisão integrativa }\end{array}$ & Revisão integrativa & 2016 & Brasil & $\begin{array}{l}\text { Inglês } \\
\text { Português }\end{array}$ \\
\hline Noronha DD, et al. & $\begin{array}{l}\text { Qualidade de vida relacionada à saúde entre adultos e } \\
\text { fatores associados: um estudo de base populacional }\end{array}$ & $\begin{array}{l}\text { Transversal } \\
\text { analítico }\end{array}$ & 2016 & Brasil & $\begin{array}{l}\text { Inglês } \\
\text { Português }\end{array}$ \\
\hline
\end{tabular}

Fonte: Rodrigues TQ, et al., 2019.

REAS/EJCH | Vol.12(4) | e2833 | DOI: https://doi.org/10.25248/reas.e2833.2020 Página 5 de 8 
Por meio da amostra identificou-se que a DA possui forte impacto na qualidade de vida dos idosos portadores, apresentando como consequência predominante a redução da autonomia e a dependência para as atividades diárias, fator este que afeta simultaneamente a vida de indivíduos responsáveis pelos cuidados daqueles que os necessitam.

\section{DISCUSSÃO}

A partir dos resultados obtidos durante as buscas, foram identificadas duas categorias principais, oriundas do corpus da análise e agrupamento: 1) Aspectos fisiopatológicos e epidemiológicos da Doença de Alzheimer; 2) Qualidade de vida e sua relação com a família e o cuidador de idosos com Doença de Alzheimer.

\section{Aspectos fisiopatológicos e epidemiológicos da Doença de Alzheimer}

A Doença de Alzheimer é a mais comum das demências acometidas na população idosa. Apresenta-se por ser um transtorno neurodegenerativo progressivo com deterioração cognitiva e de memória, comprometendo a execução das atividades básicas. Em geral, ocorre comprometimento da memória recente tornando o indivíduo incapaz de armazenar novas informações, enquanto que as lembranças remotas são preservadas até a fase acentuada da doença. Ademais, outras funções são prejudicadas como capacidade de fala, mobilidade, redução motora severa e capacidade de fazer cálculos (INOUYE K, et al., 2010).

As causas do surgimento desta doença estão associadas a perda das sinapses cerebrais causando morte neural e atrofia do cérebro. Isso acontece devido ao acúmulo de proteínas beta-amilóide que se agrupam impedindo as sinalizações nervosas e iniciando um processo inflamatório. Sabe-se que quando ocorre destruição de uma sinapse causada pelo acúmulo de beta-amilóide o cérebro aumenta ainda mais a produção desta proteína, provocando a morte de várias outras células de modo a aumentar o comprometimento neurológico do paciente até a ocorrência de sintomas mais graves. Associado a estes fatores, ocorre a fosforilação da proteína tau que formam emaranhados neurofibrilares dentro dos neurônios acarretando em comprometimento cognitivo severo (SERENIKI A, VITAL MABF, 2008).

Devido a fisiopatologia da doença é possível observar uma redução expressiva na qualidade de vida dos idosos portadores, uma vez que a perda de memória dificulta o vínculo social, afetivo e familiar, além de promover uma sobrecarga emocional e física aos cuidadores. As alterações cognitivas causadas pela DA desencadeiam vários sentimentos tanto no idoso quanto em seus cuidadores, como a impotência, desamparo, fragilidade e falta de perspectiva para o futuro, além da perda de autonomia para cuidar de si e permitir as relações sociais e familiares (INOUYE K, et al., 2010).

\section{Capacidade funcional e qualidade de vida em idosos com a Doença de Alzheimer e seus cuidadores}

As alterações relacionadas a qualidade de vida em idosos com demências variam de acordo com a evolução da doença, com diferentes níveis de comprometimento na execução segura das atividades de autocuidado e ou de maior complexidade. Geralmente as atividades funcionais de maior complexidade como por exemplo: tomar conta do próprio dinheiro e da medicação, conseguir ir sozinho a lugares distantes sem ajuda, são as primeiras funções que são perdidas ou esquecidas (BURLÁ C, et al., 2014).

Com a progressão da doença, as atividades básicas de autocuidado como: tomar banho, ir ao banheiro, trocar de roupa, ou mesmo ter continência urinária são funções esquecidas e que irão necessitar de um cuidador supervisionando a execução da mesma, ou mesmo realizar pelo idoso acometido pela Doença de Alzheimer (BURLÁ C, et al., 2014).

A capacidade funcional e a perda da autonomia entre idosos com Doença de Alzheimer podem estar relacionadas a presença da bradicinesia e sedentarismo, que associados a falta de estímulo e da ausência de uma vida ativa, favorecem a maiores chances do desenvolvimento da tríade, queda-fratura-dependência, o que leva a muitos idosos a diminuírem sua qualidade de vida (ASSIS CRC e CAMACHO ACLF, 2016).

As limitações funcionais provenientes da evolução da demência irão também trazem sofrimento ao cuidador, pessoa que se vê sobrecarregada em relação ao cuidado. Um dos principais sentimentos é a 
angústia, pois este cuidador assumiu a responsabilidade por cuidados básicos para a manutenção da vida de uma outra pessoa, e percebe que estas limitações são progressivas, sem perspectivas de qualquer melhoria (FREITAS ICC, et al., 2008).

\section{Qualidade de vida e sua relação com a família e o cuidador de idosos com Doença de Alzheimer}

Os termos saúde e qualidade de vida apesar de apresentarem suas especificidades, têm algo em comum, como a contribuição da saúde para a qualidade de vida de indivíduos ou populações, e a contribuição de muitos componentes da vida social para uma boa qualidade de vida, a fim de se alcançar um perfil elevado de saúde (PEREIRA EF, et al., 2012).

A assistência na pessoas com Doença de Alzheimer deve ser realizada por uma equipe multiprofissional e todos deverão estar capacitados para o cuidado tanto ao doente como para o cuidador, pois há uma sobrecarga para quem assume o papel de cuidar, visto que com o avançar da doença, o idoso apresenta dificuldades para a realização das tarefas da vida diária, além de alterações de se expressar verbalmente, o que compromete a relação e suas necessidades e frequentemente realiza ações inaceitáveis, devendo, portanto, a equipe multiprofissional repassar as orientações e conscientização adequadas, com vistas a uma assistência eficiente e humanizada (HOLANDA ITA, et al., 2012).

A redução da qualidade de vida pode estar intrinsecamente ligada à presença de quadro depressivo. Perda de autonomia, participação social, redução motora e comprometimento de fala e memória são fatores preponderantes para o surgimento de depressão nestes indivíduos, que passam a depender sobremaneira de terceiros para a realização de atividades básicas da vida (VITAL TM, et al., 2010). Qualidade de vida deve ser considerada como a percepção do indivíduo e sua inserção na vida, bem como no contexto de sua cultura e expectativas, padrões e preocupações atuais, de acordo com a Organização Mundial da Saúde (OMS) (NORONHA DD, et al., 2016). Dentre os diversos fatores preponderantes para a redução da qualidade de vida destes idosos a perda de autonomia se caracteriza como uma das condições mais afetadas, uma vez que progressivamente o indivíduo perde sua capacidade de viver de modo independente devido ao comprometimento cognitivo e funcional que a doença acarreta (ASSIS CRC e CAMACHO ACLF, 2016).

A maior proporção dos cuidadores não apresenta informações e nem suporte para os cuidados destinados a pacientes com DA. Tão pouco possuem conhecimentos acerca da doença e sua progressão, podendo acarretar em desgaste físico e emocional. Essa falta de informação pode acentuar o quadro depressivo do idoso reduzindo expressivamente sua qualidade de vida (FREITAS ICC, et al., 2008). A frequência de depressão nos idosos com DA variam entre $5 \%$ a $23 \%$ e esses indivíduos apresentam maior dificuldade na execução de atividades diárias, bem como maiores chances de apresentarem episódios de agitação, representando mais riscos e gerando maior estresse ao cuidador (VITAL TM, et al., 2010). Dito isso, considera-se importante reduzir as chances do surgimento da depressão em portadores da DA, fato este que pode ser minimizado a partir da implementação de exercícios físicos em sua rotina diária, além da garantia de uma maior interação social e autonomia em suas atividades cotidianas.

No curso da DA, a autonomia, é algo perdido pelo indivíduo. Quando esta ocorre há perda da autonomia, a angústia torna-se maior por parte de quem cuida. Assim aqueles que cuidam acabam por assumir a responsabilidade de fazer pelo outro, com o intuito de maximizar a preservação da individualidade e favorecer a manutenção dos direitos e vontades de quem está cuidando. Mas é preciso preparo e organização por parte da família a fim de manter as necessidades e vontades já manifestadas pelo idoso com DA (BURLÁ C, et al., 2014).

Ressalta-se a necessidade de políticas públicas efetivas voltadas para os portadores de demências, doença de Alzheimer ou de Parkinson. A implantação de programas multiprofissionais para 0 atendimento das necessidades do doente e de seus familiares, bem como, ações específicas de educação em saúde, terapias que possibilite o retardamento das limitações cognitivas e psicomotoras (HOLANDA ITA, et al., 2012). Além disto, profissionais da geriatria e a gerontologia, precisam estar cada vez mais preparados para atender e responder ao desafio para os cuidados com as demências. E precisam estar preparados não somente quanto aos cuidados físicos e sociais, mas também se atentando aos cuidados 
éticos com este idoso com DA, a fim de potencializar a integração do idoso com DA e toda a essência de um cuidado humanizado (BURLÁ C, et al., 2014).

\section{CONSIDERAÇÕES FINAIS}

Frente a elevada prevalência das doenças neurodegenerativas progressivas que acometem a população idosa foi possível observar, por meio da presente revisão de literatura, que ocorre uma redução expressiva da qualidade de vida dos idosos portadores da Doença de Alzheimer, relacionadas sobremaneira a perda de autonomia e dependência em suas atividades diárias, todos os artigos ressaltaram a diminuição da QV de quem cuida deste idoso, no caso cuidadores familiares ou formais. É fundamental para dispor de uma melhor qualidade de vida que familiares e cuidadores sejam orientados e saibam o curso progressivo da doença, de modo a agir estimulando o idoso cognitivamente e funcionalmente, estimulando-o para que possa ter maior independência para suas atividades de vida diária, em todas as fases de evolução da DA.

\section{REFERÊNCIAS}

1. ASSIS CRC, CAMACHO ACLF. Qualidade de vida dos idosos com doença de alzheimer: uma revisão integrativa. Rev enferm UFPE on line. 2016; Recife, 10(Supl. 4):3631-45.

2. BORGHI AC, et al. Qualidade de vida de idosos com doença de alzheimer e de seus cuidadores. Rev. Gaúcha Enferm. 2011; 32(4): 751-758.

3. BURLÁ C., et al. Envelhecimento e doença de Alzheimer: reflexões sobre autonomia e o desafio do cuidado. Rev. Bioét. 2014; 22(1): 85-93.

4. CESÁRIO VAC, et al. Estresse e qualidade de vida do cuidador familiar de idoso portador da doença de Alzheimer. Saúde em Debate. 2017; 41(112):171-182.

5. DAWALIBI NW, et al. Fatores relacionados à qualidade de vida de idosos em programas para a terceira idade. Ciênc. saúde coletiva. 2014: 19(8): 3505-3512.

6. FERNANDES JSG, ANDRADE MS. Revisão sobre a doença de alzheimer: diagnóstico, evolução e cuidados. Psic., Saúde \& Doenças. 2017; 18(1): 131-140.

7. FERREIRA OGL, et al. Envelhecimento ativo e sua relação com a independência funcional. Texto contexto - enferm. 2012; $21(3):$ 513-518.

8. FREITAS ICC, et al. Convivendo com o portador de Alzheimer: perspectivas do familiar cuidador. Rev. bras. enferm. 2008: 61(4): 508-513.

9. HOLANDA ITA, et al. Idosos com Alzheimer: um estudo descritivo. Rev. Rene. 2012; 13(3):582-89.

10. INOUYE K, et al. Influência da doença de Alzheimer na percepção de qualidade de vida do idoso. Rev. esc. enferm. USP. 2010; 44(4): 1093-1099.

11. MIRANDA GMD, et al. O envelhecimento populacional brasileiro: desafios e consequências sociais atuais e futuras. Rev. bras. geriatr. gerontol. 2016; 19(3): 507-519.

12. NORONHA DD, et al. Qualidade de vida relacionada à saúde entre adultos e fatores associados: um estudo de base populacional. Ciênc. Saúde coletiva. 2016; 21(2): 463-474.

13. OPAS, BRASIL. 2017. In: Demência: número de pessoas afetadas triplicará nos próximos 30 anos.

14. PEREIRA EF, et al. Qualidade de vida: abordagens, conceitos e avaliação. Rev. bras. educ. fís. esporte, São Paulo, 2012; 26(2): 241-250.

15. PEREIRA LSM, SOARES SM. Fatores que influenciam a qualidade de vida do cuidador familiar do idoso com demência. Ciência \& Saúde Coletiva. 2015; 20(12):3839-3851.

16. PEREIRA MG, GALVÃO TF. Etapas de busca e seleção de artigos em revisões sistemáticas da literatura. Epidemiol. Serv. Saúde. 2014; 23(2): 369-371.

17. SERENIKI A, VITAL MABF. A doença de Alzheimer: aspectos fisiopatológicos e farmacológicos. Rev. psiquiatr. Rio Gd. Sul. 2008; 30(1 Suppl).

18. SOARES CB, et al. Revisão integrativa: conceitos e métodos utilizados na enfermagem. Rev. esc. enferm. USP. 2014; Apr [cited 2019 Dec 27]; 48(2): 335-345.

19. VITAL TM, et al. Atividade física sistematizada e sintomas de depressão na demência de Alzheimer: uma revisão sistemática. J. bras. psiquiatr. 2010; 59(1): 58-64. 